\title{
Use of multiple 16S rRNA-targeted fluorescent probes to increase signal strength and measure cellular RNA from natural planktonic bacteria
}

\author{
SangHoon Lee ${ }^{1, *}$, Christian Malone ${ }^{2}$, Paul F. Kemp ${ }^{1}$ \\ ${ }^{1}$ Oceanographic and Atmospheric Sciences Division, Brookhaven National Laboratory, Upton, New York 11973, USA \\ ${ }^{2}$ Biology Dept, Albertson College of Idaho, Caldwell, Idaho 83605, USA
}

\begin{abstract}
Fluorescent oligonucleotide probes can be used to identify bacterial cells by hybridizing to specific rRNA sequences that are unique to a taxon. It has been suggested that single-cell RNA content could be estimated from the resulting cell fluorescence, which is directly proportional to rRNA content. However, this possibility was never assessed quantitatively for use in field studies, where most natural bacteria, due to their small size and slow growth, do not have enough rRNA to yield sufficient fluorescence for detection. One approach for increasing the fluorescence per rRNA molecule is the use of multiple probes targeted to independent sites in the rRNA molecule. In this study, we hybridized multiple probes ( 3 universal and 4 bacterial) to cultured marine bacterial isolates, and to natural bacterial assemblages from coastal water. For 5 of the 7 probes ( 2 probes did not hybridize to some of the isolates), cell fluorescence increased linearly with the number of probes hybridized. The natural bacterial assemblages showed an asymptotic increase of the probe-labeled fraction from ca $20 \%$ (1 probe) to $75 \%$ ( 5 probes) of the total population. The estimated detection limit was $0.3 \mathrm{fg}$ RNA cell ${ }^{-1}$ with 5 probes. RNA contents estimated from the fluorescence of probe-labeled cells agreed well to those determined separately by ethidium bromide fluorometry (differed by average $16 \%, n=3$ ). Calibration of the photometer against fluorescence standards was critical for accurate measurements and comparisons.
\end{abstract}

Comparisons of the base sequence of $16 \mathrm{~S}$ rRNA molecules among various taxa revealed that there are regions either relatively conserved or more variable at different levels of phylogeny (Woese 1987, Olsen 1988). The regions where base sequences are unique to a taxonomic group offer suitable target sites for molecular probing. Fluorescently labeled, $16 \mathrm{~S}$ rRNAtargeted oligonucleotide probes, when hybridized to

- Present address Polar Research Center, Korea Ocean Research and Development Institute, Ansan PO Box 29, Seoul 425-600, Korea such target sequences, can help identify individual cells by fluorescence microscopy (DeLong et al. 1989). The fluorescent probe method provides a powerful tool to study the phylogeny of naturally occurring bacterial cells (DeLong et al. 1989, Amann et al. 1990b, 1991, Distel et al. 1991). This method does not require prior culturing of cells for identification, thus avoiding the problem that few natural marine bacteria appear to be cultivable (Lee \& Fuhrman 1991).

In addition to phylogeny study, RNA content of individual cells can also be estimated from the fluorescence of the hybridized probes (DeLong et al. 1989). This is possible because, ideally, probe molecules hybridize in 1:1 proportion to cellular rRNA molecules, and each probe molecule carries a constant fluorochrome label. Since the majority of cellular RNA is ribosomal, and $16 \mathrm{~S}$ rRNA is a relatively constant fraction of the total rRNA (Bremer \& Dennis 1987), probe fluorescence should be proportional to the total RNA content of cells. DeLong et al. (1989) demonstrated a close relationship between the cellular RNA content and the fluorescence of Escherichia coli cells hybridized with fluorescent probes.

Unlike cells from lab cultures or nutrient-rich natural environments, natural marine planktonic bacteria often do not contain sufficient rRNA to generate a measurable level of fluorescence due to their small size and slow growth. To increase fluorescence, one might use a probe labeled with multiple fluorochrome molecules, or a secondary-fluorescence approach in which multiple fluorochromes (or other marker molecules) are carried to a hybridized probe by an accessory molecule. Both approaches are under development. Unfortunately, probes with multiple fluorochromes directly attached can result in poor or nonspecific binding (Amann et 
al. 1990a, DeLong 1990, Zarda et al. 1991). Secondary attachment of fluorochromes can enhance signals significantly (DeLong 1990, Zarda et al. 1991, and references therein), but the large size of the carrier molecule presents problems in delivering fluorochromes to the hybridized probes across cell walls. Procedures required for the secondary-fluorescence methods (e.g. treatment with lysozyme for the cell wall digestion, or quantitative labeling of the carrier molecule with multiple fluorochromes) seem impractical or impossible when working with a natural community of heterogenous bacterial populations.

An alternative means of increasing fluorescence is the use of multiple probes carrying single identical fluorochromes (1 fluorochrome molecule per probe), but targeted to independent sites in the rRNA molecule (Amann et al. 1990a). Using this approach, Amann et al. (1990a) observed a general increase of the fluorescence in hybridizations of 3 probes to a cultured bacterium. The multiple-probe method, if there are sufficient target sites available for a defined taxon, seems straightforward and technically simple. However, the multiple-probe method has never been quantitatively evaluated for fluorescence-based measurements of single-cell RNA content, nor tested with field samples of natural bacteria.

Cellular RNA content is known to reflect cell activities and growth rate. Past studies have shown strong correlations between the growth rates of cultured, fastgrowing bacteria and their cellular RNA contents measured from nucleic acid preparations (Schaechter et al. 1958, Bremer \& Dennis 1987, DeLong et al. 1989, Kemp et al. 1993). The correlation results from the direct role of ribosomes in protein synthesis. Given this correlation, probe-based measurements of single-cell RNA contents should prove extremely useful for the study of in situ activities of natural bacteria. Potential advantages include: (1) the method does not require sample incubations that often perturb the natural systems (Kroer \& Coffin 1992); (2) cellular RNA content of particular organisms in the mixed populations can be measured (and the organisms simultaneously identified) by using probes designed for specific organisms; and (3) measurements from individual cells will provide cell-based informations, in particular, frequency distributions. Current methods for bacterial activity measurements, which invariably average the activity over the entire assemblage, cannot provide such information.

This study explored the potential of the multipleprobe method for measuring RNA content of natural bacterioplankton. Various combinations of 7 different probes 3 universal and 4 bacterial (formerly eubacterial; Woese et al. 1990)] were hybridized to cultured or natural bacterial cells. We examined the hybridization performances of the individual probes and the increase in fluorescence with multiple probes. Cell fluorescence was converted to cellular RNA content using an empirically-determined relationship between the two. We then compared the mean RNA cell ${ }^{-1}$ in field samples, as measured independently by ethidium bromide fluorometry and probe fluorescence. They were in good agreement.

Materials and methods. Cell preparation: Cultured cells: Four marine bacterial strains, isolated from oceanic water ( 3 isolates) or sediment ( 1 isolate), were grown separately in an autoclaved seawater-based medium at $22^{\circ} \mathrm{C}$. Cells were collected on a $0.2 \mu \mathrm{m}$ Nuclepore filter (Costar, Cambridge, MA, USA), and immediately resuspended in $1 \mathrm{ml}$ of an ice-cold mixture $(9: 1$ by vol) of phosphate buffered saline solution (PBS: $145 \mathrm{mM}$ sodium chloride, $100 \mathrm{mM}$ sodium phosphate, $\mathrm{pH} \mathrm{7.5)} \mathrm{and} \mathrm{formalin} \mathrm{(filtered} \mathrm{through} \mathrm{GS} \mathrm{filter;}$

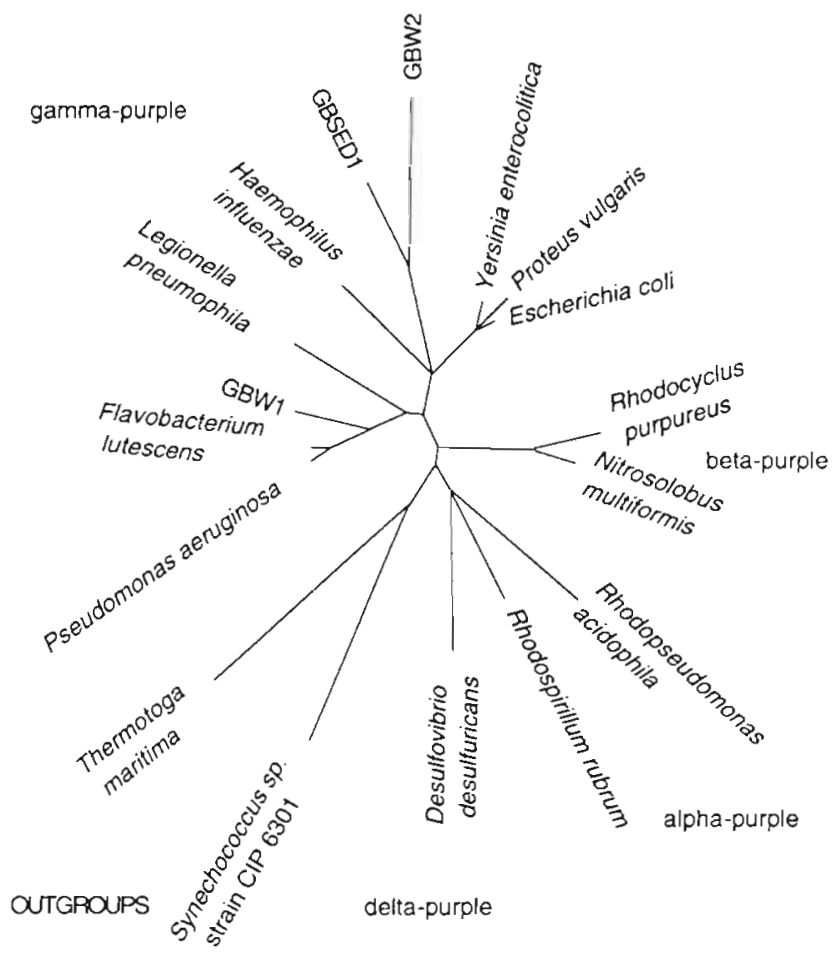

Fig. 1. Phylogenetic tree based on 165 rRNA sequence analysis. The 4 marine bacterial isolates used in this study are GBW1, GBW2, GBSED1 and SARW1; names as mnemonics for the collection locations (GB: Georges Bank; SAR: Sargasso

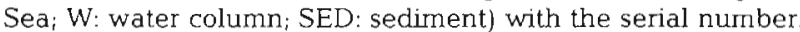
The isolates were sequenced by the reverse transcription method (Lane et al. 1988). Sequences of the other bacterial species are from the U.S. National Ribosomal RNA Database at Indiana University. The tree was constructed using the Phylip program, and shows unrooted phylogenetic distances. SARW1 was not included because the obtained sequence was not long enough for a valid comparison. Under the given growth conditions, the isolates were short rods, and the long axis was about 2 to $3 \mu \mathrm{m}$ (GBW2, GBSED) or 1 to $1.5 \mu \mathrm{m}$ (GBW1, SARW1) 
slides in $5 \times \mathrm{SET}$. The slides were washed 3 times by immersion in $0.2 \times \mathrm{SET}$ at $37^{\circ} \mathrm{C}$ for $10 \mathrm{~min}$ each and dried at room temperature. A cover slip was placed on the cell smear with a few drops of mountant ( 9 volumes of glycerol mixed with 1 volume of PBS).

Multiple probing: Probes Universal B and Eco790 did not hybridize to some of the bacterial isolates. Different combinations of the 7 probes were hybridized to the bacterial isolates, excluding any nonhybridizing probe(s) for each isolate. For the natural samples, a total of 1 to 5 probes (Universal B and Eco 790 were not used) were hybridized. For the combinations, probes were selected randomly without regard for their hybridization performance (i.e. brighter or dimmer fluorescence when used as single probes; see 'Results and discussion').

Microphotometry and epifluorescence microscopy for measurement of single-cell fluorescence: Slides were viewed with Nikon DM580 (TR) and DM400 (DAPI) excitation filters. Fluorescence was measured with a scanning microphotometer (Optical Technology Devices, Elmsford, NY, USA) mounted on a Nikon Optiphot microscope, at an emission wavelength of $600 \mathrm{~nm}$ (TR) with an Osram HBO $100 \mathrm{~W} / 2$ mercury lamp. The photometer system outputs a voltage signal which is read by an analog: digital converter board, and data are recorded automatically by a microcomputer. About 150 cells were measured from each slide.

Photometer calibration was done by measuring fluorescence of TR-coated microbeads $(n=150)$ from a standard slide (Flow Cytometry Standards Corp., Research Triangle Park, NC, USA) at every session of fluorescence measurement. Fluorescence intensities measured from cells were expressed as fractions or multiples of the mean fluorescence of the standard microbead. This provided a correction for day-to-day variations in photometer instrument settings and output.

TR fluorescence fades as the fluorochrome is exposed to irradiation, reaching to $1 / 2$ brightness after ca $35 \mathrm{~s}$. Variation of the fluorescence measurements due to photobleaching was controlled by measuring the fluorescence consistently in the first 7 to $10 \mathrm{~s}$ of the exposure.

Cultured cells were readily visible when hybridized with 1 probe. However, some natural cells were not visible even with 5 probes. The fraction of cells labeled with fluorescent probes was determined by counting DAPI-fluorescent cells and TR-fluorescent cells in the same microscope field ( $>10$ fields per slide) of the dual-labeled cells. The probe-labeled fraction is the number of TR-fluorescent cells as a percentage of DAPI-fluorescent cells.

From control slides where no probes were hybridized, we occasionally observed auto-fluorescent cells (ca $<5 \%$ of the total cell count), mostly cyanobacteria, in the natural samples. They were readily identified by their fluorescence intensity and morphology and were excluded from the TR fluorescence measurements.

DNA and RNA measurements by ethidium bromide fluorometry: Natural bacterial cells collected on the Nuclepore filters were lysed in TE $(10 \mathrm{mM}$ Tris, $1 \mathrm{mM}$ EDTA, pH 8.0) with $1 \%$ SDS. The filters were removed from the liquid, and the crude lysate was ethanolprecipitated overnight with ammonium acetate (final conc. $2.3 \mathrm{M}$ ) at $-20^{\circ} \mathrm{C}$. A crude precipitate was collected by centrifugation (10 min, $12000 \times \mathrm{g})$, dissolved in TE ( $\mathrm{pH} 8.0$ ), and extracted once with a phenol/ chloroform/isoamyl alcohol mixture $[25: 24: 1$, equilibrated with STE $(100 \mathrm{mM} \mathrm{NaCl}, 10 \mathrm{mM}$ Tris, $1 \mathrm{mM}$ EDTA, pH 8.0)], and once with chloroform. The interphase between the aqueous phase and the phenol mixture was carried to the chloroform extraction, in order to minimize the loss of nucleic acid. The final aqueous phase was again ethanol-precipitated, and the precipitate was collected by centrifugation as described above. The pellet was then dissolved in TE (pH 8.0).

Nucleic acids were quantified by a modified method of ethidium bromide fluorometry (Thoresen et al. 1983) with a Perkin Elmer spectrofluorometer (excitation at $300 \mathrm{~nm}$, emission at $600 \mathrm{~nm}$ ). DNA was measured from subsamples digested with DNase-free RNase (Boehringer Mannheim, Indianapolis, IN, USA) for $2 \mathrm{~h}$ at $37^{\circ} \mathrm{C}$. Complete digestion of RNA under these conditions was verified by agarose gel electrophoresis. RNA-fluorescence was determined as the difference in fluorescence before and after the RNase digestion. DNA - or RNA-fluorescence was converted to DNA- or RNA-amount (wt) using standard curves generated with calf thymus DNA (Sigma) or Escherichia coli rRNA (Sigma). Mean RNA cell ${ }^{-1}$ was calculated by dividing the total amount of the extracted RNA by the total number of cells collected for the nucleic acid sample.

In order to estimate the loss (or degradation) of nucleic acids during the extractions, known amounts of the standard DNA and RNA were added to parallel samples and quantified as for the natural samples. Average RNA recovery was $86 \%$, ranging from 76 to $100 \%$. DNA recovery was slightly lower $(81 \%)$. However, our DNA and RNA data were not corrected with these recovery efficiencies.

Results and discussion. In working with multiple probes, a compromise set of hybridization conditions (i.e. stringency) must be used, but the resulting hybridization efficiencies may not be identical among probes for the reasons discussed below. We first examined whether the hybridization stringency was 
optimal for all 7 probes. In general, nonspecific binding indicates low stringency, whereas higher stringencies favor perfect matches between probe-target base pairs. When checked with the negative-control probes at various concentrations (50 to $200 \mathrm{ng}$ in $30 \mu$ l of the hybridization solution), nonspecific binding was not observed under our hybridization conditions. Positive probes at elevated concentrations (100 to $200 \mathrm{ng}$ of 1 probe in $30 \mu \mathrm{l}$ ) did not increase the hybridization signal over the normal concentration (50 ng in $30 \mu \mathrm{l}$ ). Hybridization efficiencies of multiple probes under a single stringency will be further discussed below.

For all 4 bacterial isolates, fluorescence per cell increased in a linear fashion with the number of probes hybridized (Fig. 2). Each data point of Fig. 2 is a 'grand mean' obtained from 2 to 5 determinations of the mean cell fluorescence, and each determination of the mean cell fluorescence was from ca 150 cells hybridized with either single probe (single-probe hybridizations) or

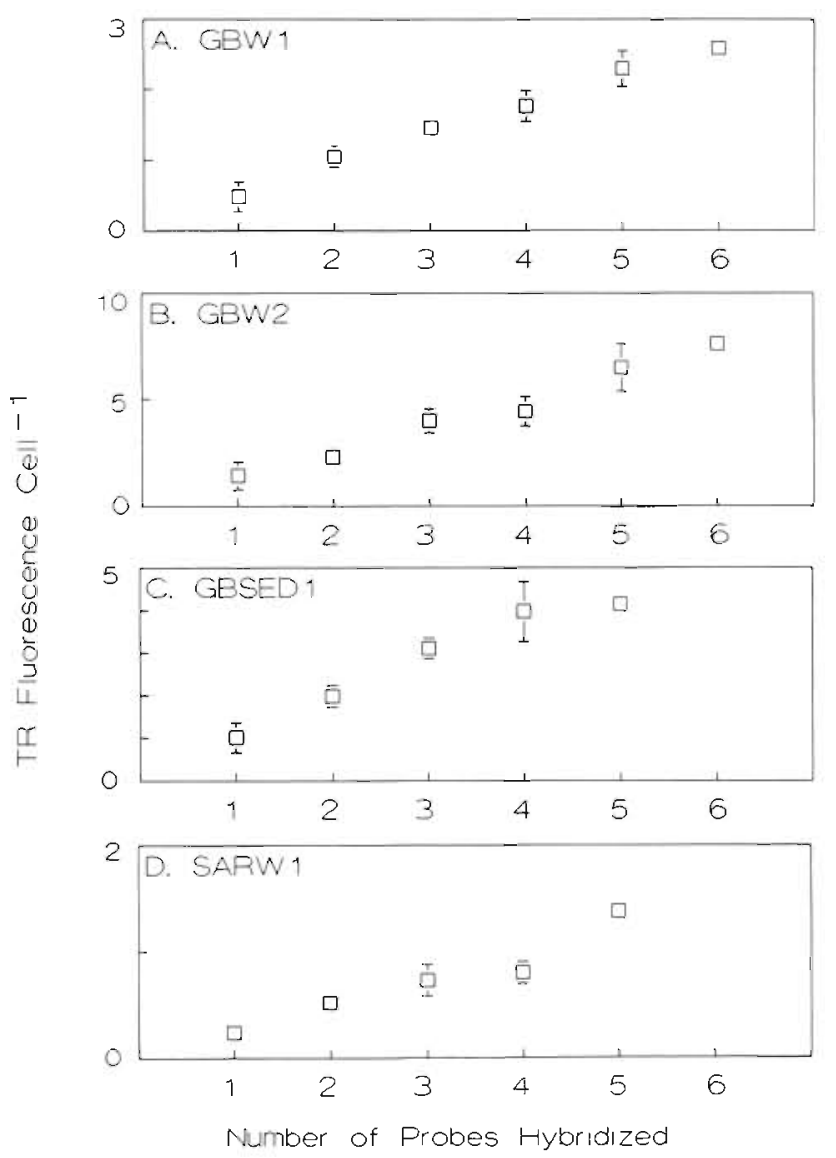

Fig. 2. Increase of mean cell fluorescence with the number of probes hybridized. Four panels from the 4 cultured marine bacterial isolates GBW1, GBW2, GBSED1 and SARW1 (see Fig. 1 for names). Each data point represents a mean from 2 to 5 determinations of mean cell fluorescence, and each determination is from ca 150 cells from a slide. Error bar: $\pm 1 \mathrm{SD}$ different combinations of the probes (multiple-probe hybridizations). Note the different scales of the cell fluorescence among the 4 isolates (Fig. 2) because of the inherent differences in cell size and cellular RNA content. For comparisons of the probe performance among isolates, we proportionately adjusted the fluorescence data for each of the 4 isolates, so that the inherently different starting points were brought to a single baseline.

For each isolate, the grand mean from the singleprobe hybridizations (the first data point in each panel of Fig. 2) was the norm for the adjustment. We divided (normalized) the mean cell fluorescence obtained from any single-probe or multiple-probe hybridizations by the grand mean of the single-probe hybridizations. The grand mean of any isolates became 1 by definition when normalized (divided by itself), providing the same baseline for comparisons across the isolates. Therefore, the normalized fluorescence is a measure of the 'performance' of any single probe or combination of probes, relative to the average performance of single probes.

In Fig. 3, individual data points are presented showing how the normalized fluorescence data are dispersed in relation to the number of probes hybridized. The pattern of the increase was nearly additive. At 4 to 5 probes, observed data appears to fall slightly (by almost $10 \%$ ) below the expected additive increase of fluorescence (dotted line). We do not exclude the possibility that probe-probe interactions may have occurred with combinations of multiple probes. For example, multiple probes may form cross-hybrids to

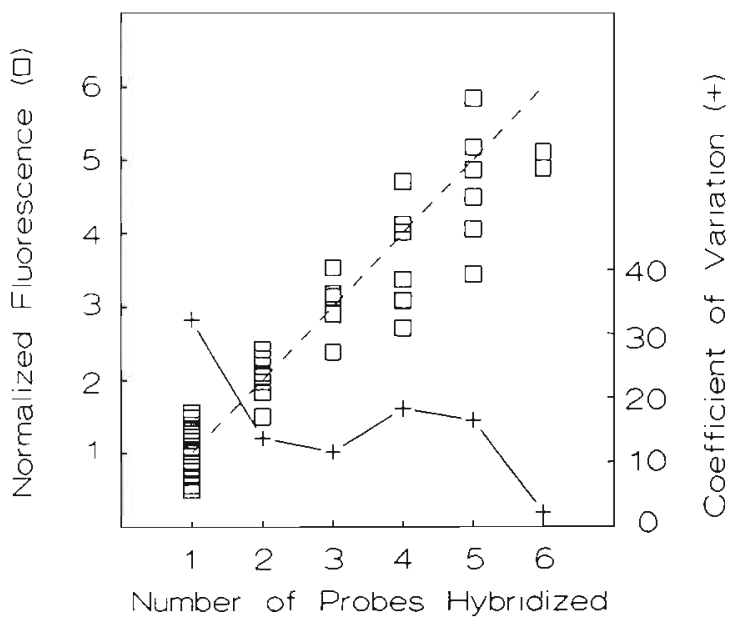

Fig. 3. Normalized mean cell fluorescence of the 4 marine bacterial isolates. See text for description of normalization. Each data point represents a mean from ca 150 cells from a slide. Dotted line shows the 1:1 ratio increase between the fluorescence and the number of probes, which represents perfect additivity of the multiple probes 
each other, resulting in improperly elevated or reduced signals. Careful selection of probe sequences will minimize this problem. However, the observed near-additive response suggests that such interactions were almost absent or minor with combinations of 2 to 5 probes. We only have 2 data points for 6 probe hybridization, because 2 probes did not bind some of the isolates.

Fluorescence of the single-probe hybridizations showed a substantial variation, ranging from ca 0.5 to 1.5 in normalized units (Fig. 3). This means the singleprobe performance varied maximally by as much as 3 times. Possible reasons for the variation are (1) experimental and instrumental errors, (2) different hybridization performances of the probes for the different bacterial isolates, i.e. probe-isolate interactions, and (3) variations in the specific fluorescence of the probes (mean fluorescence per probe molecule). Although fluorochrome-labeled oligonucleotides are routinely purified, incomplete purification or degradation during storage could cause different probes to have different specific fluorescence. However, this was found to be not significant (see below).

As for the measurement errors, (1), replicate slide preparations showed CV (coefficient of variation) of ca $10 \%$ for the mean cell fluorescence, and replicate means from a slide showed $\mathrm{CV}<10 \%$. It is unlikely that the variation in probe performance was due primarily to experimental and instrumental errors.

To evaluate the other possible reasons, (2) and (3), we examined the probe performance of the individual probe-isolate pairs. In using multiple probes under 1 stringency condition, probes of different molar $\mathrm{G}+\mathrm{C}$ content or different length number of bases) can have different optimum hybridization temperatures (Britten \& Davidson 1985), because of differences in their melting temperatures $\left(T_{\mathrm{mi}}\right.$ Table 1). Fig. 4 shows that there was no isolate that consistently hybridized well or poorly for all of the probes, and no probe yielded consistently high or low fluorescence for all of the isolates. No significant differences of the normalized fluorescence were found among isolates or probes (2-way ANOVA analysis). From the ANOVA, we concluded that variations in specific fluorescence among probes, (3), were not significant.

The ANOVA analysis indicates that probes of higher $\mathrm{G}+\mathrm{C}$ content (or higher $T_{\mathrm{m}}$ ) performed no better than probes of lower $\mathrm{G}+\mathrm{C}$ content (or lower $T_{\mathrm{m}}$ ). However, Fig. 4 shows that certain probe-isolate pairs yielded relatively low or high fluorescence, i.e. (2) probe-isolate interactions. This is probably because of the minor base-pair (1 or 2) mismatches (Amann et al. 1990b), which was unavoidable when using multiple probes against multiple target organ-

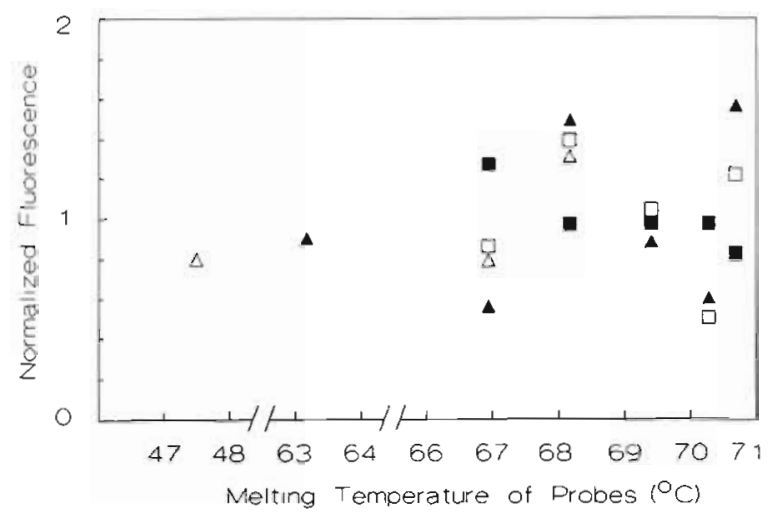

Fig. 4. Performance of the individual probe-isolate pairs. Normalized mean cell fluorescence of a probe is presented above the probe's melting temperature $\left\{T_{\mathrm{m}}\right\}$. See text for description of normalization. Each data point represents a mean of ca 150 cells from a slide. Different symbols indicate the 4 bacterial isolates. The 2 probes at the 2 highest $I_{\mathrm{m}}$ values actually have the same $T_{\mathrm{m}}\left(70.3^{\circ} \mathrm{C}\right)$, but are separated for clarity

isms under a single stringency. Structural conformation of an rRNA molecule may also affect hybridization efficiency, restricting access to the target sites, especially for in situ hybridizations (Taneja \& Singer 1987). Both of these problems equally apply to the method using a probe labeled with multiple fluorochromes, however it is more difficult to assess the mechanism or the impact of reduced hybridization efficiency when single-fluorochrome-labeled multiple probes are used.

Although the single-probe performances varied substantially among the probe-isolate pairs (3-fold; Fig. 3), or within a probe (2-fold; Fig. 4), performances of multiple probes showed less variation with use of multiple probes. CV decreased with increasing number of probes (Fig. 3). We speculate this is due to the overall signal enhancement (increase of mean), as well as the use of multiple probes which probably dampened the variation of individual probe's performance by adding up the positive or negative deviations from the mean (decrease of variance). Use of multiple probes thus should give more reliable estimates of RNA content in natural mixed populations of bacteria, compared to any single probe.

Unlike the cultured marine isolates, natural bacterial assemblages contained cells that were not visibly probe-labeled. The fraction of the probe-labeled cells increased asymptotically with increasing number of probes (Fig. 5). Combinations of 3 or 4 probes labeled most of the cells that could be readily detected by the current technique; the labeled fraction increased only slightly with 4 or 5 probes. Use of still more probes would not substantially improve labeling with the current state of the fluorochrome technique and micro- 


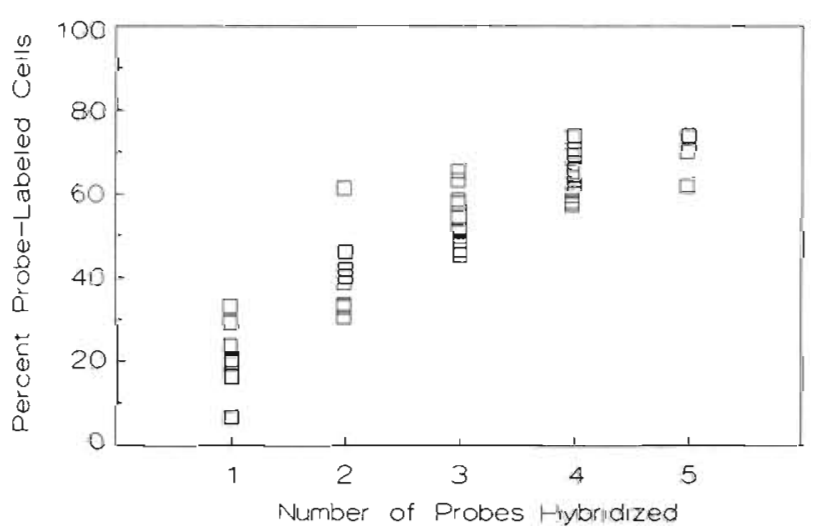

Fig. 5. Percentage of probe-labeled natural bacterial cells increased with the number of probes hybridized. Data from 3 natural coastal samples (February 27, March 10 and March 24, 1992). Each data point represents mean of $>10$ fields from a slide. Total populations were determined by DAPI direct count (Porter \& Feig 1980) and the probe-labeled cells are presented as \% of the total

scope optics. This suggests that about 3 to 5 times signal enhancement by any other method would be satisfactory for labeling those 'detectable' cells.

Even with 5 probes, ca $25 \%$ of the natural cells were not labeled. We currently have little information regarding whether those unlabeled cells lacked accessible target sites for any of the probes used, or are impermeable to the probes, or (most likely) they do not have enough RNA to be detected by our current procedures. Dormant cells, for example, may have extremely low RNA content. Most of the unlabeled cells were small cocci common in seawater, although some cells did not fluoresce despite their large cell size. Very small cells may contain undetectable amounts of cellular RNA even if they are metabolically active, simply because of their small size. For this reason, bacterial cells from oceanic environments, where nutrients are in lower concentrations and smaller cells are predominant, may have a higher percentage of cells that are not detectable.

We calculated the mean cell fluorescence of the natural populations by averaging the fluorescence from the probe-labeled cells over the entire population, which includes the cells detectable with DAPI but not with probe fluorescence. This is equivalent to recording zero fluorescence for the undetectable cells. It was necessary for a correct comparison of the fluorescence-based RNA to the RNA content determined by ethidium bromide (EtBr) fluorometry, which averages the RNA over the entire population. As with the cultured marine isolates, cell fluorescence of the natural samples increased in a linear and additive fashion with the number of probes hybridized (Fig. 6).

We converted the mean cell fluorescence to cellu-
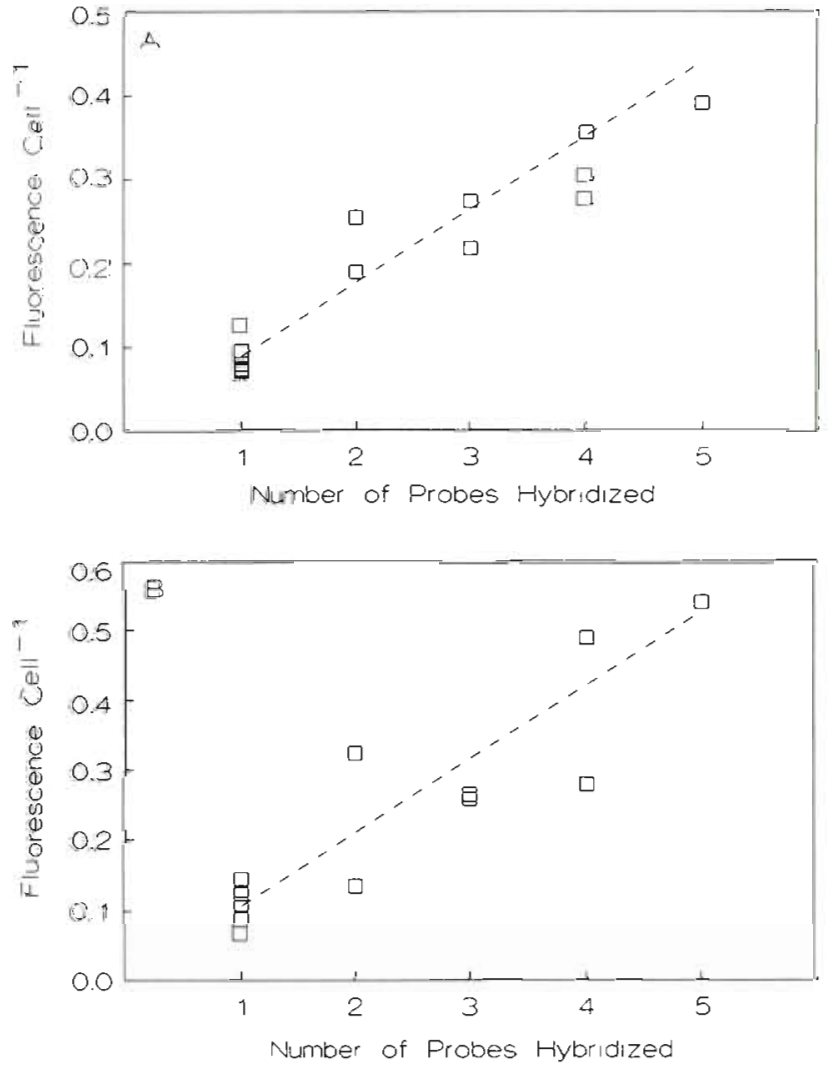

Fig. 6. Increase of the mean cell fluorescence of natural bacterial cells with increasing number of probes hybridized. Each data point is from ca 150 cells from a slide. Dotted line shows the $1: 1$ ratio increase between the fluorescence and the number of probes, which represents the perfect additivity of the multiple probes. (A) Sample collected February 27, 1992. (B) Sample collected March 10, 1992

lar RNA content, using an empirical relationship (regression; $\mathrm{n}=17, \mathrm{r}^{2}=0.79$ ) obtained from a separate study (authors' unpubl. data) where cell fluorescence was related to cellular RNA determined by the EtBr fluorometry, i.e. the same method used in this study. The RNA contents determined by the 2 independent methods, probe fluorescence and $\mathrm{EtBr}$ fluorometry, agreed well (Fig. 7). They differed by an average of $16 \%$. This supports the quantitative use of the fluorescent probe approach for in situ studies of RNA contents, as a tool for examining microbial activity.

This technique measures femtograms of RNA from individual natural bacterial cells (Fig. 7). Mean RNA cell ${ }^{-1}$ reported in this study, 3 to $5 \mathrm{fg}$, agrees well with the cellular RNA content of marine bacteria calculated from the cell size using a conversion factor (Simon \& Azam 1989, and references therein). The average signal of the cell fluorescence (equivalent to 3 to $5 \mathrm{fg}$ RNA) was typically more than 10 times the background noise of the photometer. Therefore, we esti- 


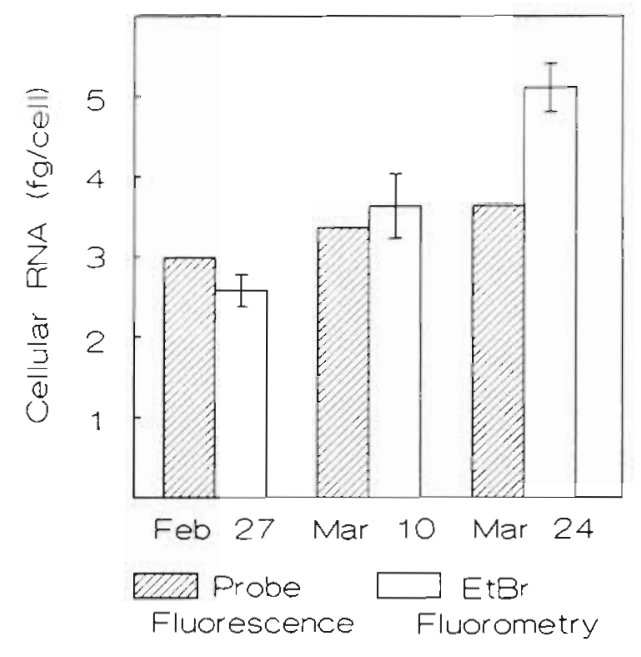

Fig. 7. Comparisons of cellular RNA contents estimated from cell fluorescence (from 5-probe hybridizations, unreplicated data) to the RNA contents measured by ethidium bromide (EtBr) fluorometry (mean of 2 or 3 replicates). Error bar: $\pm 1 \mathrm{SD}$

mated 0.3 to $0.5 \mathrm{fg}$ cell $^{-1}$ for the lowest RNA content detectable by probe fluorescence $\left(<0.3 \mathrm{fg} \mathrm{cell}^{-1}\right.$ for the unlabeled natural cells).

Although strong correlations between growth rate and cellular RNA content have been found in laboratory studies (Schaechter et al. 1958, Bremer \& Dennis 1987, DeLong et al. 1989, Kemp et al. 1993), further studies are needed for application and interpretation of RNA content measurements in the field. For example, one must consider how to convert RNA content to cell activities (e.g. growth rate), or what factors would affect the relationships between the RNA content and the cell activity. If this technique is used for a particular species of bacteria in a natural assemblage, it may be possible to convert the RNA content to a growth rate using a relationship empirically determined in the laboratory. However, for a natural assemblage that contains diverse bacterial species, cellular RNA. contents may prove difficult to interpret (as activity measurement) because of the inherent differences among species. Simultaneous measurements of cellular RNA content, DNA content, or size in individual cells by flow cytometry or image analysis, may help correct for these inherent differences. The method presented here allows us to begin examining the usefulness of RNA-frequency distributions.

Acknowledgements. We thank Edward DeLong for providing helpful information and guidance, Julie LaRoche for valuable discussions and critical review, Jeneen Wagner for help with lab work, and anonymous reviewers for their criticism. This work was supported by the U.S. Department of Energy under Contract No. DE-AC02-76CH00016.

\section{LITERATURE CITED}

Amann, R. I., Binder, B. J., Olson, R. J., Chisholm, S. W., Devereux R., Stahl, D. A. (1990a). Combination of $16 \mathrm{~S}$ rRNA-targeted oligonucleotide probes with flow cytometry for analyzing mixed microbial populations. Appl. environ. Microbiol. 56: 1919-1925

Amann, R. I., Krumholz, L., Stahl, D. A. (1990b). Fluorescentoligonucleotide probing of whole cells for determinative. phylogenetic and environmental studies in microbiology. J. Bact. 172: 762-770

Amann, R., Springer, N., Ludwig, L., Görtz, H., Schleifer, K.-H. (1991). Identification in situ and phylogeny of uncultured bacterial endosymbionts. Nature 351. 161-164

Bremer, H., Dennis, P. P. (1987). Modulation of chemical composition and other parameters of the cell by growth rate. In: Neidhardt, F. C. (ed.) Escherichia coli and Salmonella typhimurium: cellular and molecular biology. American Society for Microbiology, Washington, DC, p. 1527-1542

Britten, R. J., Davidson, E. H. (1985). Hybridisation strategy. In: Hames, B. D., Higgins, S. J. (eds.) Nucleic acid hybridisation: a practical approach. IRL Press, Oxford, p. 3-15

DeLong, E. F. (1990). A signal amplification method for in situ hybridizations using fluorescently-labeled, rRNAtargeted probes; Abstr. R-3. Abstr. 90th A. Meet. Am. Soc. Microbiol. 1990. American Society for Microbiology, Washington, DC, p. 246

DeLong, E. F., Wickham, G. S., Pace, N. R. (1989). Phylogenetic stains: ribosomal RNA-based probes for the identification of single cells. Science 243: 1360-1363

Distel, D. L., DeLong, E. F., Waterbury, J. B. (1991). Phylogenetic characterization and in situ localization of the bacterial symbiont of shipworms (Teredinidae: Bivalvia) by using $16 \mathrm{~S}$ rRNA sequence analysis and oligodeoxynucleotide probe hybridization. Appl. environ. Microbiol. 57: $2376-2382$

Giovannoni, S. J., DeLong, E. F., Olsen, G. J., Pace, N. R. (1988). Phylogenetic group-specific oligodeoxynucleotide probes for identification of single microbial cells. J. Bacteriol. 170: 720-726

Hicks, R. E., Amann, R. I., Stahl, D. A. (1992). Dual staining of natural bacterioplankton with 4',6-diamidino-2-phenylindole and fluorescent oligonucleotide probes targeting kingdom-level 16S rRNA sequences. Appl. environ. Microbiol. 58: 2158-2163

Kemp, P. F., Lee, S., LaRoche, J. (1993). Estimating the growth rate of slowly growing marine bacteria from RNA content Appl. environ. Microbiol. 59: 2594-2601

Kroer, N., Coffin, R. B. (1992). Microbial trophic interactions in aquatic microcosms designed for testing genetically engineered microorganisms: a field comparison. Microb. Ecol. 23: 143-15?

Lane, D. J., Field, K. G., Olsen, G. J., Pace, N. R. (1988). Reverse transcriptase sequencing of ribosomal RNA for phylogenetic analysis. Meth. Enzymol. 167: 138-144

Lee, S., Fuhrman, J. A. (1991). Species composition shift of confined bacterioplankton studied at the level of community DNA. Mar. Ecol. Prog. Ser. 79: 195-201

Olsen, G. J. (1988). Phylogenetic analysis using ribosomal RNA sequences. Meth. Enzymol. 164: 793-812

Porter, K. G., Feig, Y S. (1980). The use of DAPI for identifying and counting aquatic microflora. Limnol. Oceanogr. 25: 943-948

Schaechter, E., Maaloe, O., Kjeldgaard, N. O. (1958). Dependence on medium and temperature of cell size and chemical composition during balanced growth of Salmonella typhimurium. J. gen. Microbiol. 19:592-606 
Simon, M., Azam, F. (1989). Protein content and protein synthesis rates of planktonic marine bacteria. Mar Ecol. Prog. Ser. 51: 201-213

Taneja, K., Singer, R. H. (1987). Use of oligodeoxynucleotide probes for quantitative in situ hybridization to actin mRNA. Anal. Biochem. 166: 389-398

Thoresen, S. S., Clayton, J. R. Jr, Dortch, Q., Ahmed, S. I (1983). A rapid technique for the determination of RNA and DNA in marine phytoplankton. J. Plankton Res. 5 $253-261$

This note was presented by E. and B. Sherr, Corvallis, Oregon, USA
Woese, C. R. (1987). Bacterial evolution. Microbiol. Rev. 51: 221-271

Woese, C. R., Kandler, O., Wheelis, M. L. (1990). Towards a natural system of organisms: Proposal for the domains Archaea, Bacteria, and Eucarya. Proc. natl Acad. Sci. U.S.A. 87: $4576-4579$

Zarda, B., Amann, R., Wallner, G., Schleifer, K.-H. (1991). Identification of single bacterial cells using digoxigeninlabelled, rRNA-targeted oligonucleotides. J gen. Microbiol. 137: 2823-2830

Manuscript first received: November 17, 1992

Revised version accepted: July 20, 1993 